\title{
Major depressive disorder in patients with diabetes mellitus in Pietermaritzburg, South Africa
}

\author{
J M Jansen van Vuuren, ${ }^{1,2} \mathrm{MB}$ BCh, MMedSc (Internal Medicine); S Pillay, ${ }^{3,4} \mathrm{PhD}$ \\ ${ }^{1}$ Postgraduate Programme (Master of Medical Science), School of Clinical Medicine, University of KwaZulu-Natal, Durban, South Africa \\ ${ }^{2}$ Pietermaritzburg Hospital Complex, KwaZulu-Natal, South Africa \\ ${ }^{3}$ Department of Internal Medicine, Edendale Hospital, Pietermaritzburg, South Africa \\ ${ }^{4}$ University of KwaZulu-Natal, Durban, South Africa
}

Corresponding author: J M Jansenvan Vuuren (juanjvanvuuren@icloud.com)

Background. Diabetes mellitus (DM) and major depressive disorder (MDD) constitute a major burden of disease. A study found that type $2 \mathrm{DM}(\mathrm{DM} 2)$ is associated with a two-fold increase in risk of developing MDD worldwide - except in Africa. The present study challenges this statement.

Objectives. To determine the prevalence of MDD in a cohort of patients with DM and its effect on glycaemic control.

Methods. A cross-sectional study was performed at a diabetes clinic at Edendale Hospital, Pietermaritzburg, South Africa (1 March 31 May 2017). A total of 176 participants were enrolled. Participants completed the Patient Health Questionnaire-9 (PHQ-9), with a score of $\geq 10$ representing a diagnosis of MDD. Higher PHQ-9 scores translate into more severe MDD. A surrogate for glycaemic control, glycated haemoglobin (HbA1c) blood testing, was also performed.

Results. The majority of participants were female and suffered from DM2. The mean age was 54.4 years. The prevalence of MDD in the study population was $46.6 \%$. A significantly positive linear relationship was shown between PHQ-9 score and HbA1c ( $p=0.018253$ ).

Conclusions. MDD appears to constitute a large burden of disease in our population of individuals with DM. A relationship between the severity of the MDD and HbAlc suggests that prompt intervention in both conditions is likely to improve health outcomes.

S Afr Med J 2019;109(1):58-61. DOI:10.7196/SAMJ.2019.v109i1.13356

Diabetes mellitus (DM) is a common medical condition in both the developed and developing worlds, affecting $>420$ million people worldwide. ${ }^{[1]}$ It is projected that type $2 \mathrm{DM}$ (DM2) will have a global prevalence of $7.7 \%$ (439 million people) by the year $2030 .{ }^{[2]}$ Major depressive disorder (MDD) too is a disorder affecting a large proportion of the population. A national epidemiological survey by Hasin et al. ${ }^{[3]}$ showed that MDD represents a heavy burden of disease, with a lifetime prevalence of $13.2 \%$ in a North American population. The lifetime prevalence of MDD in South Africa (SA) is said to be $9.7 \%{ }^{[4]}$

Numerous studies conducted in the past decade show a definite association between DM2 and MDD, the former increasing the risk of the latter and vice versa. ${ }^{[5-9]}$ In 2010 , a systematic review showed that patients with DM2 have a $24 \%$ increased risk of developing MDD compared with those without DM2. ${ }^{[10]}$ According to a study analysing individuals from 47 countries published in 2013, DM2 is associated with a two-fold increase in risk of developing MDD worldwide - except in Africa. ${ }^{[11]}$ Mommersteeg et al. ${ }^{[11]}$ noted that their study had several limitations and cited underdiagnosis, cultural differences and variability of the reliability of self-reported depressive symptoms as potential reasons for their findings. Their conclusion was challenged by a group of researchers in Zambia, who showed that major depressive symptoms were common in the Zambian population with DM2. ${ }^{[12]}$ Although research concerning individuals with type $1 \mathrm{DM}$ (DM1) has focused mainly on young patients, an increased prevalence of MDD was also found in these individuals. ${ }^{[13]}$ It is well known that DM increases cardiovascular disease risk, as an independent variable and as part of a group of disorders. Patients who have both DM2 and MDD have a combined two- to three-fold increased risk of cardiovascular disease, especially strokes. ${ }^{[14]}$ Van der Feltz-Cornelius et al. ${ }^{[15]}$ concluded that good glycaemic control requires attention to be directed towards both DM and MDD.

It can be extrapolated from the above that comorbid MDD in individuals with DM poses a significant public health issue. Shrestha et al. ${ }^{[16]}$ published data highlighting its economic impact, with substantial increases in health expenditure documented, while Hay et al. ${ }^{[17]}$ showed the cost benefits of a collaborative management approach in patients with both DM and MDD. Data on the prevalence and effect of MDD in South Africans appear to be limited.

\section{Objectives}

To sensitise clinicians to and shed light on the potentially lifethreatening combination of DM and MDD by determining: (i) the prevalence of MDD; and (ii) the effect of MDD on glycaemic control in a group of patients attending a specialised diabetes clinic in Pietermaritzburg, SA.

\section{Methods}

This article forms part of research for the purposes of a thesis. In an observational, cross-sectional study, voluntary adult participants who attended a specialised diabetes clinic at Edendale Hospital, a 900-bed public sector, urban regional hospital in Pietermaritzburg, were consecutively sampled over a period of 3 months (1 March - 31 May 2017). All consenting adult patients with DM1 and DM2 were considered for inclusion in the study. Adolescents were excluded. The sample size required at a type 1 error of $5 \%$ was 135 , assuming the hypothesis is correct that the prevalence of major depressive symptoms is not higher in patients in Africa. ${ }^{[1]}$ 
Each participant was allocated a number and their age, sex, diabetes type, Patient Health Questionnaire-9 (PHQ-9) score and laboratory reference number for the glycated haemoglobin $(\mathrm{HbAlc})$ test were recorded for analysis on a standardised clinical sheet. In addition, certain potential confounders were recorded for analysis: hypothyroidism, epilepsy, HIV and body mass index (BMI).

Participants were presented with the PHQ-9 in either English or isiZulu. The PHQ-9 is a well-developed, reliable screening tool for MDD, with a score of $\geq 10$ having a sensitivity of $88 \%$ and a specificity of $88 \%$ for the diagnosis of MDD, with higher scores translating into increasing severity of MDD. ${ }^{[18]}$ The PHQ-9 was validated by Bhana et al. ${ }^{[19]}$ in 2015 for use in SA on chronic care patients. They used a score of $\geq 9$ to diagnose MDD. When compared with the Patient Health Questionnaire-2 and the Structured Clinical Interview for DSM-IV, the sensitivity and specificity of the PHQ-9 were $49 \%$ and $94 \%$, respectively. Despite its considerably lower sensitivity, Bhana et al. ${ }^{[19]}$ determined that the PHQ-9 can be a valuable tool for identifying comorbid depression. The PHQ-9 was validated in isiZulu for the purposes of our study.

HbAlc ( $\mathrm{mmol} / \mathrm{mol}$ ) blood results from samples obtained on the day of consultation were recorded (for conversion into HbAlc percentage, see Table 1). HbAlc is a reference system developed by the International Federation of Clinical Chemistry to standardise $\mathrm{HbAlc}$ reporting worldwide. The local National Health Laboratory Service used the Bio-Rad D10 machine (Bio-Rad, South Africa) to determine HbAlc values.

The protocol and all tools needed for this study were presented for assessment to the Biomedical Research Ethics Committee of the University of KwaZulu-Natal. Full approval was obtained (ref. no. BE483/16).

Statistical analyses were performed to determine the prevalence of MDD and

Table 1. Conversion table for HbAlc from $\mathrm{mmol} / \mathrm{mol}$ to $\%$

\begin{tabular}{ll}
\hline HbAlc (mmol/mol) & HbAlc (\%) \\
\hline 31 & 5 \\
42 & 6 \\
53 & 7 \\
64 & 8 \\
75 & 9 \\
86 & 10 \\
97 & 11 \\
108 & 12 \\
HbAlc = glycated haemoglobin.
\end{tabular}

simple regression was calculated using the Pearson correlation coefficient.

\section{Results}

\section{Demographics}

Data for a total of 176 participants were analysed. Their mean age was 54.4 (standard deviation (SD) 14.8) years (range 19 - 96); $125(71.0 \%)$ were female and $51(29.0 \%)$ male; and 20 (11.4\%) had DM1 and 151 (85.8\%) had DM2 (DM type was not recorded for the remaining 5 participants). The mean PHQ-9 score was 9.26 (SD 5.67).

No relationship was found between age and PHQ-9 score $(p=0.90)$, and no difference in PHQ-9 score was evident between female and male participants $(p=0.44)$.

\section{Prevalence of MDD in participants with DM}

A total of 82 participants scored $\geq 10$ on the PHQ-9 and were diagnosed with MDD. The prevalence of MDD in patients with DM was therefore $46.6 \%$.

\section{HbAlc and MDD in DM}

The mean HbAlc value was 76.89 (SD 25.26) $\mathrm{mmol} / \mathrm{mol}$.

A positive, statistically significant relationship was shown between the PHQ-9 score and HbA1c ( $p=0.018253$ ) (Fig. 1).

No statistically significant difference in HbAlc was found between participants diagnosed with MDD (mean 80.0; 95\% confidence interval (CI) 74.0 - 86.0) and those not diagnosed with MDD (mean 74.1; 95\% CI 69.5 - 78.7) $(p=0.1226)$.

\section{Analysis of potential confounders}

A total of 170 participants had all the variables required for statistical analysis of the potential confounders recorded.
A total of 6 participants were known to have hypothyroid disease. Compared with participants without hypothyroidism, no statistical difference in PHQ-9 score was evident between the two groups ( $p=0.65$ ).

A total of 12 participants were known to have epilepsy. Compared with participants without comorbid epilepsy, no statistical difference in PHQ-9 score was evident between the two groups $(p=0.82)$.

A total of 35 participants were known to be HIV-positive. Compared with participants who were HIV-negative, no statistical difference in PHQ-9 score was evident between the two groups ( $p=0.54$ ).

There was no statistically significant relationship between BMI and PHQ-9 score $(p=0.69)$.

\section{Discussion}

Our study population consisted of patients attending a specialised diabetes clinic in a 900-bed public sector, urban regional hospital in Pietermaritzburg, SA. The majority of participants were female, which concurs with the finding of a study performed at the same clinic in 2015 that $>77 \%$ of clinic attendees were female. ${ }^{[20]}$ There was no difference in PHQ-9 score between our female and male participants $(p=0.44)$.

The mean age of participants was 54.4 years, and no relationship was evident between age and PHQ-9 score $(p=0.90)$. According to the Society for Endocrinology, Metabolism and Diabetes of South Africa's 2017 guidelines, ${ }^{[21]}$ the recommended age at which to start screening for depression in addition to cognitive impairment and dementia in patients with diabetes, with a level B evidence, is 65 years. Our study suggests that the younger population could require screening, and this requires further research.

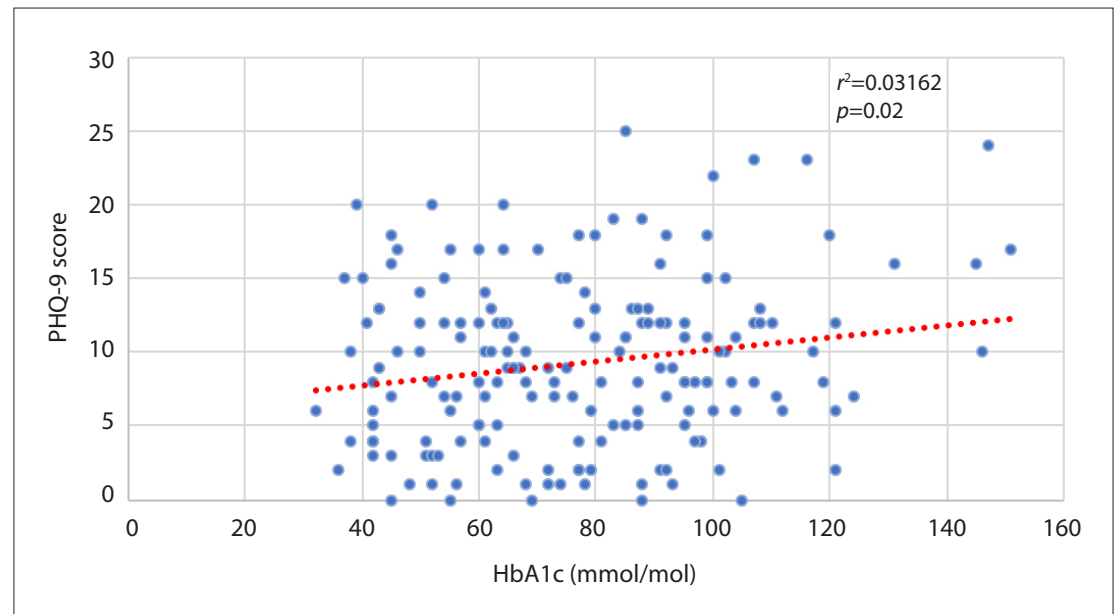

Fig. 1. Scatter diagram illustrating the linear relationship between HbAlc and PHQ-9 scores. (HbAlc $=$ glycated haemoglobin; PHQ-9 = Patient Health Questionnaire-9.) 
The prevalence of MDD in the general population of SA is $9.7 \% .^{[4]}$ This is far lower than the $46.6 \%$ prevalence of MDD among our study participants. If the cut-off for the diagnosis of MDD of a PHQ-9 score $\geq 9$ had been used, as in the study by Bhana et al., ${ }^{[19]}$ the prevalence would have been $51.1 \%$. This difference of $4.6 \%$ requires further research to ascertain its clinical significance.

A significantly positive linear relationship was shown between the PHQ-9 score and HbAlc ( $p=0.018253)$. HbAlc is a surrogate marker for glycaemic control. This association provides evidence that poor glycaemic control is related to higher PHQ-9 scores, and therefore more severe MDD. As this was a cross-sectional study, causal relationships could not be established; however, the finding remains significant, and, although further studies are required, assists in providing evidence in favour of screening for and treating MDD in patients with DM. Poor glycaemic control in patients with DM and MDD has been described, and depression has been shown to worsen the course of chronic conditions. ${ }^{[22,23]}$ To suggest a possible solution, a biopsychosocial approach must be utilised. The hypothalamic-pituitary axis plays a large role in the pathophysiology of depressive disorders, with elevated levels of corticotropin-releasing factor found in the cerebrospinal fluid of patients with depression and anxiety. ${ }^{[24]}$ Together with increased levels of noradrenaline, this results in elevated cortisol. ${ }^{[25]}$ Cortisol is diabetogenic, and an elevated cortisol level will result in elevated serum glucose, in turn resulting in poorer glycaemic control. ${ }^{[26]}$ The reward pathway in the brain may also play a role. Increased consumption of certain food types, notably carbohydrates, which are associated with the release of dopamine, and chocolate, which contains andamines that promote elevated mood, is common in patients with depression. ${ }^{[27]}$ This selfperpetuating cycle results in poorer glycaemic control, as many of these foods result in acute elevation of serum glucose. Psychological conditions influence the use of diabetes medication and increase the prevalence of poor treatment adherence. ${ }^{[28]}$ This potential influence may be attributed to factors such as altered help-seeking behaviour due to avolition and feelings of worthlessness. The prevalence of MDD has been found to be higher in rural areas compared with urban areas. ${ }^{[29]}$ Patients in rural areas may have difficulty in collecting their diabetes medication and following up at their local clinics, possibly accounting for the above association. Although our study took place in a regional hospital in a periurban setting, the diabetes clinic drains patients from the second most populous health district in KZN, the uMgungundlovu district, of which rural areas form part.

Potential confounders were analysed in an attempt to negate their effect on the study results. There were no differences in PHQ-9 score when comparing participants without with participants known to have the confounders hypothyroidism $(p=0.65)$, epilepsy $(p=0.82)$ or HIV $(p=0.54)$. In addition, no relationship was shown between PHQ-9 score and BMI $(p=0.69)$. It would appear that these groups of participants were equally affected by symptoms of MDD and that these confounders imposed no additional risk.

\section{Study limitations}

As this was an observational, cross-sectional study, it had many intrinsic limitations. Causal inference was difficult to establish, and it will remain a challenge in determining which condition preceded the other. A distinction was not made between MDD and other mood disorders. The symptoms and signs of MDD may be caused by other conditions, such as hypothyroidism (although this was analysed). ${ }^{[30]}$ These conditions were excluded clinically by the treating clinicians, and exclusion was highly dependent on their clinical expertise. The diagnosis of depressive disorder due to another medical condition (in this instance DM) could account for the depressive symptoms. It is difficult to distinguish between MDD and depressive disorder due to another medical condition and, for simplicity, we did not attempt to do this. ${ }^{[31]}$ Other potential confounders, notably level of education, smoking, substance use and psychosocial support, were not recorded.

\section{Conclusions and recommendations}

Early diagnosis of MDD is of paramount importance in patients with DM. Cardiovascular risk increases markedly in patients with both DM and MDD. ${ }^{[14]}$ It has been concluded that patients with both DM and MDD are at an increased risk of complications related to diabetes as well as early death, and that optimisation of treatment is required for optimal patient outcome. ${ }^{[32-34]}$

The findings of the present study suggest that MDD does in fact constitute a problem among individuals with $\mathrm{DM}$ in an African setting, with $46.6 \%$ of patients diagnosed with MDD. A surrogate for the severity of MDD (the PHQ-9 score) has a linear relationship with measured HbAlc, a surrogate for the control of diabetes. Although more studies are required to validate the findings of this study in populations in other settings in Africa, routine screening for MDD in patients with diabetes is recommended.

It is hoped that the findings of this study will sensitise clinicians to the risk of MDD in patients with DM and the potential effect MDD may have on their glycaemic control and overall wellbeing.

Declaration. This research was a requirement for JMJvV's Master of Medical Sciences degree.

Acknowledgements. None.

Author contributions. The manuscript was derived from data analysed by JMJvV. SP was the supervisor for this degree.

Funding. None.

Conflicts of interest. None.

1. Roglic G, World Health Organization. Global Report on Diabetes. Geneva: WHO, 2016.

2. Shaw JE, Sicree RA, Zimmet PZ. Global estimates of the prevalence of diabetes for 2010 and 2030 Shaw JE, Sicree RA, Zimmet PZ. Global estimates of the prevalence of diabetes for
Diabetes Res Clin Pract 2010;87(1):4-14. https://doi.org/10.1016/j.diabres.2009.10.007

3. Hasin DS, Goodwin RD, Stinson FS, Grant BE. Epidemiology of major depressive disorder: Results from the National Epidemiologic Survey on Alcoholism and Related Conditions. Arch Gen Psychiatry 2005;62(10):1097-1106. https://doi.org/10.1001/archpsyc.62.10.1097

4. Tomlinson M, Grimsrud AT, Stein DJ, Williams DR, Myer L. The epidemiology of major depression in South Africa: Results from the South African stress and health study. S Afr Med J 2009;99(5 Pt 2):367-373.

5. Mezuk B, Heh V, Prom-Wormley E, Kendler KS, Pedersen NL. Association between major depression and type 2 diabetes in midlife: Findings from the Screening Across the Lifespan Twin Study. Psychosom Med 2015;77(5):559-566. https://doi.org/0.1097/PSY.0000000000000182

6. Foran E, Hannigan A, Glynn L. Prevalence of depression in patients with type 2 diabetes mellitus in Irish primary care and the impact of depression on the control of diabetes. Ir J Med Sci 2015;184(2):319322. https://doi.org/10.1007/s11845-014-1110-7

7. Mezuk B, Chen Y, Yu C, et al. Depression, anxiety, and prevalent diabetes in the Chinese population: Findings from the China Kadoorie Biobank of 0.5 million people. J Psychosom Res 2013;75(6):511517. https://doi.org/10.1016/j.jpsychores.2013.09.008

8. Ali S, Stone MA, Peters JL, Davies MJ, Khunti K. The prevalence of co-morbid depression in adults with type 2 diabetes: A systematic review and meta-analysis. Diabet Med 2006;23(11):1165-1173. https://doi.org/10.1111/j.1464-5491.2006.01943.x

9. Myers AK, Grannemann BD, Lingvay I, Trivedi MH. Brief report: Depression and history of suicide attempts in adults with new-onset type 2 diabetes. Psychoneuroendocrinology 2013;38(11):2810-2814 https://doi.org/10.1016/.jpsyneuen.2013.06.013

10. Nouwen A, Winkley K, Twisk J, et al. Type 2 diabetes mellitus as a risk factor for the onset of depression: A systematic review and meta-analysis. Diabetologia 2010;53(12):2480-2486. https://doi. org/10.1007/s00125-010-1874-X

11. Mommersteeg PM, Herr R, Pouwer F, Holt RI, Loerbroks A. The association between diabetes and an episode of depressive symptoms in the 2002 World Health Survey: An analysis of 231,797 individuals episode of depressive symptoms in the 2002 World Health Survey: An analysis of 231,797
from 47 countries. Diabet Med 2013;30(6):e208-e214. https://doi.org/10.1111/dme.12193

12. Hapunda G, Abubakar A, Pouwer F, van de Vijver F. Diabetes mellitus and comorbid depression in Hapunda G, Abubakar A, Pouwer F, van de Vijver F. Diabetes mellitus and
Zambia. Diabet Med 2015;32(6):814-818. https://doi.org/10.1111/dme.12645

13. Kovacs M, Goldston D, Obrosky DS, Bonar LK. Psychiatric disorders in youths with IDDM: Rates and Kovacs M, Goldston D, Obrosky DS, Bonar LK. Psychiatric disorders in youths with IDDV
risk factors. Diabetes Care 1997;20(1):36-44. https://doi.org/10.2337/diacare.20.1.36

14. Ting RZ, Lau ES, Ozaki R, et al. High risk for cardiovascular disease in Chinese type 2 diabetic patients with major depression - a 7-year prospective analysis of the Hong Kong Diabetes Registry. J Affect Disord 2013;149(1-3):129-135. https://doi.org/10.1016/j.jad.2013.01.012

15. Van der Feltz-Cornelis CM, Nuyen J, Stoop C, et al. Effect of interventions for major depressive disorder and significant depressive symptoms in patients with diabetes mellitus: A systematic review and meta-analysis. Gen Hosp Psychiatry 2010;32(4):380-395. https://doi.org/10.1016/j. genhosppsych.2010.03.011

16. Shrestha SS, Zhang P, Li R, Thompson TJ, Chapman DP, Barker L. Medical expenditures associated with major depressive disorder among privately insured working-age adults with diagnosed diabetes in the United States, 2008. Diabetes Res Clin Pract 2013;100(1):102-110. https://doi.org/10.1016/j. diabres.2013.02.002 
17. Hay JW, Katon WJ, Ell K, Lee PJ, Guterman JJ. Cost-effectiveness analysis of collaborative care management of major depression among low-income, predominantly Hispanics with diabetes. Value Health 2012;15(2):249-254. https://doi.org/10.1016/.j.jval.2011.09.008

18. Kroenke K, Spitzer RL, Williams JB. The PHQ-9: Validity of a brief depression severity measure. J Gen Intern Med 2001;16(9):606-613. https://doi.org/10.1046/j.1525-1497.2001.016009606.x

19. Bhana A, Rathod SD, Selohilwe O, Kathree T, Petersen I. The validity of the Patient Health Questionnaire for screening depression in chronic care patients in primary health care in South Africa. BMC Psychiatry 2015;15:118. https://doi.org/10.1186/s12888-015-0503-0

20. Pillay S, Aldous C, Mahomed F. Diabetic patients served at a regional level hospital: What is their clinical picture? J Endocrinol Metab Diabetes S Afr 2015;20(1):50-56. https://doi.org/10.1080/16089 677.2015.1030856

21. Society for Endocrinology, Metabolism and Diabetes of South Africa Type 2 Diabetes Guideline Expert Committee. Chapter 24: Type 2 diabetes in older persons in 2017 SEMDSA Guideline for the Management of Type 2 Diabetes Guideline Committee. JEMDSA 2017; 22(1)(Suppl 1):S1-S196.

22. Papelbaum M, Moreira RO, Coutinho W, et al. Depression, glycemic control and type 2 diabetes. Diabetol Metab Syndr 2011;3(1):26. https://doi.org/10.1186/1758-5996-3-26

23. Simon GE. Treating depression in patients with chronic disease: Recognition and treatment are crucial; depression worsens the course of a chronic illness. West J Med 2001;175(5):292-293.

24. Arborelius L, Owens MJ, Plotsky PM, Nemeroff CB. The role of corticotropin-releasing factor in Arborelius L, Owens MJ, Plotsky PM, Nemeroff CB. The role
depression and anxiety disorders. J Endocrinol 1999;160(1):1-12.

25. Hughes JW, Watkins L, Blumenthal JA, Kuhn C, Sherwood A. Depression and anxiety symptoms are related to increased 24 -hour urinary norepinephrine excretion among healthy middle-aged women J Psychosom Res 2004;57(4):353-358.
26. Kollind M, Adamson U, Lins PE, Efendic S. Diabetogenic action of GH and cortisol in insulindependent diabetes mellitus: Aspects of the mechanisms behind the Somogyi phenomenon. Horm Metab Res 1987;19(4):156-159. https://doi.org/10.1055/s-2007-1011766

7. Singh M. Mood, food, and obesity. Front Psychol 2014;5:925. https://doi.org/10.3389/fpsyg. 2014.00925 27. Balhara YP. Diabetes and psychiatric disorders. Indian J Endocrinol Metab 2011;15(4):274-283. Balhara YP. Diabetes and psychiatric
https://doi.org/10.4103/2230-8210.85579

29. Probst JC, Laditka SB, Moore CG, Harun N, Powell MP, Baxley EG. Rural-urban differences in depression prevalence: Implications for family medicine. Fam Med 2006;38(9):653-660.

30. Patten SB. Long-term medical conditions and major depression in the Canadian population. Can J Psychiatry 1999;44(2):151-157. https://doi.org/10.1177/070674379904400205

31. Identifying and managing depression in the medical patient. Prim Care Companion J Clin Psychiatry 2005; 7(6):282-293; quiz 94-95

32. Holt RI, de Groot M, Golden SH. Diabetes and depression. Curr Diab Rep 2014;14(6):491. https://doi. org/10.1007/s11892-014-0491-3

33. Lloyd CE. Diabetes and depression. Curr Diab Rep 2002;2(6):465-466.

34. Katon WJ. The comorbidity of diabetes mellitus and depression. Am J Med 2008;121(11 Suppl 2):S8S15. https://doi.org/10.1016/j.amjmed.2008.09.008

Accepted 16 July 2018 